12th International Symposium on Cosmology and

Particle Astrophysics (CosPA 2015)

International Journal of Modern Physics: Conference Series

Vol. 43 (2016) 1660202 (5 pages)

(C) The Author(s)

DOI: $10.1142 / \mathrm{S} 2010194516602027$

\title{
Small Scale Isocurvature Perturbation of Weakly Interacting Massive Particle
}

\author{
Ki-Young Choi \\ Korea Astronomy and Space Science Institute, Daejeon 305-348, Korea \\ kiyoungchoi@kasi.re.kr \\ Jinn-Ouk Gong \\ Asia Pacific Center for Theoretical Physics, Pohang, 790-784, Korea \\ Department of Physics, Postech, Pohang 790-784, Korea \\ jinn-ouk.gong@apctp.org \\ Chang Sub Shin \\ Department of Physics and Astronomy, Rutgers University, Piscataway NJ 08854, USA \\ changsub@physics.rutgers.edu \\ Published 7 July 2016
}

\begin{abstract}
It is known that the smallest size of the structures of the Universe with the weakly interacting massive dark matter is determined by the scale that enters the Hubble horizon at the time of kinetic decoupling of WIMP. This comes from the fact that the perturbation at smaller scales is erased due to the collisional damping during the kinetic decoupling. However the isocurvature mode is not affected and continue to be constant. We discuss about the generation of the isocurvature mode of WIMP dark matter at small scales recently found by Choi, Gong, and $\operatorname{Shin}^{1}$ and its implications for the indirect detection of dark matter through the formation of the small size of halos.
\end{abstract}

Keywords: WIMP; dark matter; isocurvature perturbation; structure formation.

\section{Adiabaticity of dark matter}

The weakly interacting massive particle (WIMP) has been studied as a good candidate for dark matter due to the natural determination of the relic density during the standard evolution of the big bang Universe and the viable detectability in the near future. ${ }^{2}$ In the standard WIMP scenario, they are produced from the thermal plasma by scatterings and therefore have intrinsically adiabatic density perturbation. This is consistent with the current observation from the analysis of the cosmic

This is an Open Access article published by World Scientific Publishing Company. It is distributed under the terms of the Creative Commons Attribution 4.0 (CC-BY) License. Further distribution of this work is permitted, provided the original work is properly cited. 
microwave background (CMB) temperature anisotropy, which constrains the power spectrum of the isocurvature mode between dark matter and radiation to be smaller than a few percent compared to the adiabatic one. ${ }^{3}$

WIMP is coupled to the background plasma by its small but non-negligible interactions so that they could be in the thermal equilibrium in the early Universe. Due to the expansion of the Universe, when they become non-relativistic, the interaction becomes less efficient and finally the number density in the comoving volume is fixed, that is so called WIMP freeze-out. The resulting relic density of WIMP becomes naturally the same order of that for dark matter. ${ }^{4,5}$ However even after the freeze-out of the chemical equilibrium, the kinetic interactions are still active to make dark matter tightly coupled to the background plasma by exchanging the energy and momentum.

Finally the elastic scattering which maintains the kinetic equilibrium becomes inefficient and the dark matters are completely decoupled. During the kinetic decoupling process, the adiabatic mode of dark matter are erased due to the collisional damping and the structures of the smaller size hardly form. ${ }^{6-9}$

\section{Isocurvature mode at small scales}

The adiabaticity is the natural result of the single inflation model, since all the matters are produced from the single source of the inflaton decay. ${ }^{10}$ When the WIMP dark matter is produced from the thermal plasma, the dark matter is also adiabatic. Their densities satisfy the relation

$$
\delta_{m}=\frac{3}{4} \delta_{r}
$$

where $\delta \equiv \delta \rho / \rho$ for each species of dark matter and radiation.

However this adiabaticity of WIMP is applied to the super horizon modes. When the corresponding scale enters the horizon, the density of dark matter and radiation do not follow the adiabatic relation Eq.(1) any more, if they are decoupled already. While the radiation density oscillates under the competition of gravity and pressure, that of the dark matter grows only due to the gravity. Therefor the isocurvature perturbation defined as

$$
S \equiv \delta_{m}-\frac{3}{4} \delta_{r}
$$

is generated in the sub horizon scales. However this does not make any problem since the small scale isocurvature perturbation is not constrained by the observations.

For much smaller scales, they may enter the horizon when the WIMP is still kinetically coupled to the radiation. In this epoch, since both dark matter and radiation is tightly coupled, they can be considered as a single fluid and the density perturbation of the adiabatic mode oscillates together. Therefore it can be said that the dark matter is still adiabatic in the sub horizon satisfying the relation in Eq.(1). The relation begins to break down when dark matter decouples from the radiation. 
At the same time, the adiabatic modes of WIMP experiences the collisional damping during the kinetic decoupling.

However we have to note that this behaviour of the dark matter perturbation is only applied to the adiabatic mode. The isocurvature mode of dark matter does not follow the radiation perturbation and does not damped away either. ${ }^{11,12}$

\section{Generation of the isocurvature perturbation of WIMP dark matter}

The kinetic equilibrium does not imply the adiabatic density perturbation in itself, since the number density of the fluids is not changing. That is different from the chemical equilibrium, where all the isocurvature mode are erased and only adiabatic mode survives. Even though two fluids, here dark matter and radiation, are in the kinetic equilibrium, when there is a change in the number density, the isocurvature perturbation can be created. One example was shown by Choi, Gong, and Shin. ${ }^{1}$

In the letter, they considered the early matter domination before radiation dominated epoch of the standard big bang model. That usually happens in the standard inflation models during the reheating process by the oscillating inflaton field, or in the weakly interacting heavy particles which decays very late. ${ }^{13,14}$ The only condition for generating the isocurvature modes is that the reheating temperature $T_{\text {reh }}$ is lower than the (chemical) freeze-out temperature of WIMP $T_{\mathrm{fr}}$,

$$
T_{\text {reh }}<T_{\text {fr }} .
$$

Since the reheating temperature is lower, the chemical freeze-out of WIMP happens during the early matter domination epoch. Even during the epoch between the chemical decoupling and the reheating, there is continuous production of radiation from the decay of the dominating heavy particle, and the WIMPs are produced from those radiation. Therefore the source of the radiation and dark matter can be considered as the same mother particle. However after the chemical freeze-out, while the WIMP number density does not change any more, the radiation is still being generated from the decaying heavy particle. During this process, the density perturbation of dark matter and radiation have different evolution and the isocurvature perturbation can be created. This is also applied to the single inflation models.

After completing the reheating, WIMP dark matter has isocurvature mode as well as adiabatic one, as an initial condition of the radiation dominated Universe. Since WIMP is still in the kinetic equilibrium, the adiabatic mode of them behaves following the density perturbation of the radiation, oscillating and being damped. However the isocurvature mode of WIMP is just constant and do not oscillate and damped either.

\section{Implications of the isocurvature mode of WIMP}

In the standard model of WIMP dark matter, the kinetic decoupling scale gives the smallest size of the structures due to the collisional damping during the kinetic 
decoupling. However during the epoch of the early matter domination before the radiation domination, the large isocurvatue mode can be generated after chemical decoupling and not damped away during the kinetic decoupling even though its scale is smaller than the kinetic decoupling scale. The unsuppressed isocurvature mode can finally seed the small size mini halos, ${ }^{15,16}$ which was considered to be impossible in the standard WIMP model.

The existence of the small scale mini halos has many implications. The WIMP dark matter annihilation in the mini halos can enhance the signals in the indirect detection of dark matter via the gamma-ray, cosmic rays and neutrinos. ${ }^{17,18}$ The mini halos can affect the microlensing ${ }^{19-21}$ and direct detection too. ${ }^{22,23}$ The interaction of dark matter can play as warm dark matter ${ }^{24}$ to explain the discrepancy of the standard $\Lambda$ CDM model.

\section{Acknowledgments}

J.G. acknowledges the Max-Planck-Gesellschaft, the Korea Ministry of Education, Science and Technology, Gyeongsangbuk-Do and Pohang City for the support of the Independent Junior Research Group at the Asia Pacific Center for Theoretical Physics. J.G. is also supported by a Starting Grant through the Basic Science Research Program of the National Research Foundation of Korea (No. 2013R1A1A1006701). CSS is supported in part by DOE grants doe-sc0010008, DOEARRA-SC0003883, and DOE-DE-SC0007897.

\section{References}

1. K. Y. Choi, J. O. Gong and C. S. Shin, Phys. Rev. Lett. 115 (2015) 21, 211302 doi:10.1103/PhysRevLett.115.211302 [arXiv:1507.03871 [astro-ph.CO]].

2. H. Baer, K. Y. Choi, J. E. Kim and L. Roszkowski, Phys. Rept. 555 (2014) 1 doi:10.1016/j.physrep.2014.10.002 [arXiv:1407.0017 [hep-ph]].

3. P. A. R. Ade et al. [Planck Collaboration], Planck 2013 results. XVI. Cosmological parameters, Astron. Astrophys. 571 (2014) A16 [arXiv:1303.5076 [astro-ph.C0]].

4. B. W. Lee and S. Weinberg, Cosmological Lower Bound on Heavy Neutrino Masses, Phys. Rev. Lett. 39 (1977) 165.

5. P. Hut, Phys. Lett. B 69 (1977) 85. doi:10.1016/0370-2693(77)90139-3

6. C. Boehm, P. Fayet and R. Schaeffer, Phys. Lett. B 518 (2001) 8 doi:10.1016/S03702693(01)01060-7 [astro-ph/0012504].

7. S. Hofmann, D. J. Schwarz and H. Stoecker, Damping scales of neutralino cold dark matter, Phys. Rev. D 64 (2001) 083507 [astro-ph/0104173].

8. A. M. Green, S. Hofmann and D. J. Schwarz, The First wimpy halos, JCAP 0508 (2005) 003 [astro-ph/0503387].

9. T. Bringmann, Particle Models and the Small-Scale Structure of Dark Matter, New J. Phys. 11 (2009) 105027 [arXiv:0903.0189 [astro-ph.CO]].

10. S. Weinberg, Phys. Rev. D 70 (2004) 043541 doi:10.1103/PhysRevD.70.043541 [astro$\mathrm{ph} / 0401313]$.

11. P. J. E. Peebles, ApJ, 315:L73 (1987).

12. W. Hu and N. Sugiyama, Toward understanding CMB anisotropies and their implications, Phys. Rev. D 51 (1995) 2599 [astro-ph/9411008]. 
13. K. Kohri, M. Yamaguchi and J. Yokoyama, Neutralino dark matter from heavy gravitino decay, Phys. Rev. D 72 (2005) 083510 [hep-ph/0502211].

14. K. Y. Choi, J. E. Kim, H. M. Lee and O. Seto, Neutralino dark matter from heavy axino decay, Phys. Rev. D 77 (2008) 123501 [arXiv:0801.0491 [hep-ph]].

15. J. Diemand, B. Moore and J. Stadel, Earth-mass dark-matter haloes as the first structures in the early Universe, Nature 433 (2005) 389 [astro-ph/0501589].

16. V. S. Berezinsky, V. I. Dokuchaev and Y. N. Eroshenko, Small-scale clumps of dark matter, Phys. Usp. 57 (2014) 1 [Usp. Fiz. Nauk 184 (2014) 3] [arXiv:1405.2204 [astroph.HE]].

17. T. Bringmann, P. Scott, Y. Akrami and, Improved constraints on the primordial power spectrum at small scales from ultracompact minihalos, Phys. Rev. D 85 (2012) 125027 [arXiv:1110.2484 [astro-ph.CO]].

18. Y. Yang, G. Yang and H. Zong, Neutrino signals from ultracompact minihalos and constraints on the primordial curvature perturbation, Phys. Rev. D 87 (2013) 10, 103525 [arXiv:1305.4213 [astro-ph.CO]].

19. A. V. Gurevich, K. P. Zybin and V. A. Sirota, Small scale structure of dark matter and microlensing, Sov. Phys. Usp. 167 (1997) 913 [astro-ph/9801314].

20. M. Ricotti and A. Gould, A New Probe of Dark Matter and High-Energy Universe Using Microlensing, Astrophys. J. 707 (2009) 979 [arXiv:0908.0735 [astro-ph.CO]].

21. F. Li, A. L. Erickcek and N. M. Law, A new probe of the small-scale primordial power spectrum: astrometric microlensing by ultracompact minihalos, Phys. Rev. D 86, 043519 (2012) [arXiv:1202.1284 [astro-ph.CO]].

22. M. Kamionkowski and S. M. Koushiappas, Galactic substructure and direct detection of dark matter, Phys. Rev. D 77 (2008) 103509 [arXiv:0801.3269 [astro-ph]].

23. A. W. Adams and J. S. Bloom, Direct detection of dark matter with space-based laser interferometers, astro-ph/0405266.

24. C. Boehm, A. Riazuelo, S. H. Hansen and R. Schaeffer, Phys. Rev. D 66 (2002) 083505 doi:10.1103/PhysRevD.66.083505 [astro-ph/0112522]. 